\title{
A symmetric Nörlund sum with application to inequalities
}

\author{
Artur M. C. Brito da Cruz, Natália Martins and Delfim F. M. Torres \\ [Submitted 18-Oct-2011; accepted 09-March-2012; to Proceedings of International Conference on \\ Differential \& Difference Equations and Applications, in honour of Professor Ravi P. Agarwal, to \\ be published by Springer in the series Proceedings in Mathematics (PROM)]
}

\begin{abstract}
Properties of an $\alpha, \beta$-symmetric Nörlund sum are studied. Inspired in the work by Agarwal et al., $\alpha, \beta$-symmetric quantum versions of Hölder, CauchySchwarz and Minkowski inequalities are obtained.
\end{abstract}

\section{Introduction}

The symmetric derivative of function $f$ at point $x$ is defined as $\lim _{h \rightarrow 0}(f(x+h)-$ $f(x-h)) /(2 h)$. The notion of symmetrically differentiable is interesting because if a function is differentiable at a point then it is also symmetrically differentiable, but the converse is not true. The best known example of this fact is the absolute value function: $f(x)=|x|$ is not differentiable at $x=0$ but is symmetrically differentiable at $x=0$ with symmetric derivative zero [6].

Quantum calculus is, roughly speaking, the equivalent to traditional infinitesimal calculus but without limits [4]. Therefore, one can introduce the symmetric quantum derivative of $f$ at $x$ by $(f(x+h)-f(x-h)) /(2 h)$. As in any calculus, it is then natural to develop a corresponding integration theory, looking to such integral as the inverse operator of the derivative.

Artur M. C. Brito da Cruz ${ }^{1,2}$

e-mail:artur.cruz@estsetubal.ips.pt

Natália Martins ${ }^{2}$

e-mail: natalia@ua.pt

Delfim F. M. Torres ${ }^{2}$

e-mail: delfimeua.pt

${ }^{1}$ Escola Superior de Tecnologia de Setúbal, Estefanilha, 2910-761 Setúbal, Portugal

${ }^{2}$ Center for Research and Development in Mathematics and Applications

Department of Mathematics, University of Aveiro, 3810-193 Aveiro, Portugal 
The main goal of this paper is to study the properties of a general symmetric quantum integral that we call, due to the so-called Nörlund sum [4], the $\alpha, \beta$ symmetric Nörlund sum.

The paper is organized as follows. In Section 2 we define the forward and backward Nörlund sums. Then, in Section 3 , we introduce the $\alpha, \beta$-symmetric Nörlund sum and give some of its properties. We end with Section 4 proving $\alpha, \beta$-symmetric versions of Hölder's, Cauchy-Schwarz's and Minkowski's inequalities.

\section{Forward and backward Nörlund sums}

This section is dedicated to the inverse operators of the $\alpha$-forward and $\beta$-backward differences, $\alpha>0, \beta>0$, defined respectively by

$$
\Delta_{\alpha}[f](t):=\frac{f(t+\alpha)-f(t)}{\alpha}, \quad \nabla_{\beta}[f](t):=\frac{f(t)-f(t-\beta)}{\beta} .
$$

Definition 1. Let $I \subseteq \mathbb{R}$ be such that $a, b \in I$ with $a<b$ and $\sup I=+\infty$. For $f: I \rightarrow \mathbb{R}$ and $\alpha>0$ we define the Nörlund sum (the $\alpha$-forward integral) of $f$ from $a$ to $b$ by

$$
\int_{a}^{b} f(t) \Delta_{\alpha} t=\int_{a}^{+\infty} f(t) \Delta_{\alpha} t-\int_{b}^{+\infty} f(t) \Delta_{\alpha} t
$$

where $\int_{x}^{+\infty} f(t) \Delta_{\alpha} t=\alpha \sum_{k=0}^{+\infty} f(x+k \alpha)$, provided the series converges at $x=a$ and $x=b$. In that case, $f$ is said to be $\alpha$-forward integrable on $[a, b]$. We say that $f$ is $\alpha$-forward integrable over $I$ if it is $\alpha$-forward integrable for all $a, b \in I$.

Until Definition 2 (the backward/nabla case), we assume that $I$ is an interval of $\mathbb{R}$ such that $\sup I=+\infty$. Note that if $f: I \rightarrow \mathbb{R}$ is a function such that $\sup I<+\infty$, then we can extend function $f$ to $\tilde{f}: \tilde{I} \rightarrow \mathbb{R}$, where $\tilde{I}$ is an interval with $\sup \tilde{I}=+\infty$, in the following way: $\left.\tilde{f}\right|_{I}=f$ and $\left.\tilde{f}\right|_{\tilde{I} \backslash I}=0$.

Using the techniques of Aldwoah in his Ph.D. thesis [2], it can be proved that the $\alpha$-forward integral has the following properties:

Theorem 1. If $f, g: I \rightarrow \mathbb{R}$ are $\alpha$-forward integrable on $[a, b], c \in[a, b], k \in \mathbb{R}$, then

1. $\int_{a}^{a} f(t) \Delta_{\alpha} t=0$;

2. $\int_{a}^{b} f(t) \Delta_{\alpha} t=\int_{a}^{c} f(t) \Delta_{\alpha} t+\int_{c}^{b} f(t) \Delta_{\alpha} t$, when the integrals exist;

3. $\int_{a}^{b} f(t) \Delta_{\alpha} t=-\int_{b}^{a} f(t) \Delta_{\alpha} t$;

4. $k f$ is $\alpha$-forward integrable on $[a, b]$ and $\int_{a}^{b} k f(t) \Delta_{\alpha} t=k \int_{a}^{b} f(t) \Delta_{\alpha} t$;

5. $f+g$ is $\alpha$-forward integrable on $[a, b]$ and 


$$
\int_{a}^{b}(f+g)(t) \Delta_{\alpha} t=\int_{a}^{b} f(t) \Delta_{\alpha} t+\int_{a}^{b} g(t) \Delta_{\alpha} t
$$

6. if $f \equiv 0$, then $\int_{a}^{b} f(t) \Delta_{\alpha} t=0$.

Theorem 2. Let $f: I \rightarrow \mathbb{R}$ be $\alpha$-forward integrable on $[a, b]$. If $g: I \rightarrow \mathbb{R}$ is a nonnegative $\alpha$-forward integrable function on $[a, b]$, then $f g$ is $\alpha$-forward integrable on $[a, b]$.

Proof. Since $g$ is $\alpha$-forward integrable, then both series $\alpha \sum_{k=0}^{+\infty} g(a+k \alpha)$ and $\alpha \sum_{k=0}^{+\infty} g(b+k \alpha)$ converge. We want to study the nature of series $\alpha \sum_{k=0}^{+\infty} f g(a+k \alpha)$ and $\alpha \sum_{k=0}^{+\infty} f g(b+k \alpha)$. Since there exists an order $N \in \mathbb{N}$ such that $|f g(b+k \alpha)| \leqslant$ $g(b+k \alpha)$ and $|f g(a+k \alpha)| \leqslant g(a+k \alpha)$ for all $k>N$, then both $\alpha \sum_{k=0}^{+\infty} f g(a+k \alpha)$ and $\alpha \sum_{k=0}^{+\infty} f g(b+k \alpha)$ converge absolutely. The intended conclusion follows.

Theorem 3. Let $f: I \rightarrow \mathbb{R}$ and $p>1$. If $|f|$ is $\alpha$-forward integrable on $[a, b]$, then $|f|^{p}$ is also $\alpha$-forward integrable on $[a, b]$.

Proof. There exists $N \in \mathbb{N}$ such that $|f(b+k \alpha)|^{p} \leqslant|f(b+k \alpha)|$ and $|f(a+k \alpha)|^{p} \leqslant$ $|f(a+k \alpha)|$ for all $k>N$. Therefore, $|f|^{p}$ is $\alpha$-forward integrable on $[a, b]$.

Theorem 4. Let $f, g: I \rightarrow \mathbb{R}$ be $\alpha$-forward integrable on $[a, b]$. If $|f(t)| \leqslant g(t)$ for all $t \in\left\{a+k \alpha: k \in \mathbb{N}_{0}\right\}$, then for $b \in\left\{a+k \alpha: k \in \mathbb{N}_{0}\right\}$ one has

$$
\left|\int_{a}^{b} f(t) \Delta_{\alpha} t\right| \leqslant \int_{a}^{b} g(t) \Delta_{\alpha} t
$$

Proof. Since $b \in\left\{a+k \alpha: k \in \mathbb{N}_{0}\right\}$, there exists $k_{1}$ such that $b=a+k_{1} \alpha$. Thus,

$$
\begin{aligned}
\left|\int_{a}^{b} f(t) \Delta_{\alpha} t\right| & =\left|\alpha \sum_{k=0}^{+\infty} f(a+k \alpha)-\alpha \sum_{k=0}^{+\infty} f\left(a+\left(k_{1}+k\right) \alpha\right)\right| \\
& =\left|\alpha \sum_{k=0}^{+\infty} f(a+k \alpha)-\alpha \sum_{k=k_{1}}^{+\infty} f(a+k \alpha)\right|=\left|\alpha \sum_{k=0}^{k_{1}-1} f(a+k \alpha)\right| \\
& \leqslant \alpha \sum_{k=0}^{k_{1}-1}|f(a+k \alpha)| \leqslant \alpha \sum_{k=0}^{k_{1}-1} g(a+k \alpha) \\
& =\alpha \sum_{k=0}^{+\infty} g(a+k \alpha)-\alpha \sum_{k=k_{1}}^{+\infty} g(a+k \alpha)=\int_{a}^{b} g(t) \Delta_{\alpha} t .
\end{aligned}
$$

Corollary 1. Let $f, g: I \rightarrow \mathbb{R}$ be $\alpha$-forward integrable on $[a, b]$ with $b=a+k \alpha$ for some $k \in \mathbb{N}_{0}$.

1. If $f(t) \geqslant 0$ for all $t \in\left\{a+k \alpha: k \in \mathbb{N}_{0}\right\}$, then $\int_{a}^{b} f(t) \Delta_{\alpha} t \geqslant 0$.

2. If $g(t) \geqslant f(t)$ for all $t \in\left\{a+k \alpha: k \in \mathbb{N}_{0}\right\}$, then $\int_{a}^{b} g(t) \Delta_{\alpha} t \geqslant \int_{a}^{b} f(t) \Delta_{\alpha} t$.

We can now prove the following fundamental theorem of the $\alpha$-forward calculus. 
Theorem 5 (Fundamental theorem of Nörlund calculus). Let $f: I \rightarrow \mathbb{R}$ be $\alpha$ forward integrable over I. Let $x \in I$ and define $F(x):=\int_{a}^{x} f(t) \Delta_{\alpha} t$. Then, $\Delta_{\alpha}[F](x)=$ $f(x)$. Conversely, $\int_{a}^{b} \Delta_{\alpha}[f](t) \Delta_{\alpha} t=f(b)-f(a)$.

Proof. If $G(x)=-\int_{x}^{+\infty} f(t) \Delta_{\alpha} t$, then

$$
\begin{aligned}
\Delta_{\alpha}[G](x) & =\frac{G(x+\alpha)-G(x)}{\alpha}=\frac{-\alpha \sum_{k=0}^{+\infty} f(x+\alpha+k \alpha)+\alpha \sum_{k=0}^{+\infty} f(x+k \alpha)}{\alpha} \\
& =\sum_{k=0}^{+\infty} f(x+k \alpha)-\sum_{k=0}^{+\infty} f(x+(k+1) \alpha)=f(x) .
\end{aligned}
$$

Therefore, $\Delta_{\alpha}[F](x)=\Delta_{\alpha}\left(\int_{a}^{+\infty} f(t) \Delta_{\alpha} t-\int_{x}^{+\infty} f(t) \Delta_{\alpha} t\right)=f(x)$. Using the definition of $\alpha$-forward difference operator, the second part of the theorem is also a consequence of the properties of Mengoli's series. Since

$$
\begin{aligned}
\int_{a}^{+\infty} \Delta_{\alpha}[f](t) \Delta_{\alpha} t & =\alpha \sum_{k=0}^{+\infty} \Delta_{\alpha}[f](a+k \alpha)=\alpha \sum_{k=0}^{+\infty} \frac{f(a+k \alpha+\alpha)-f(a+k \alpha)}{\alpha} \\
& =\sum_{k=0}^{+\infty}(f(a+(k+1) \alpha)-f(a+k \alpha))=-f(a)
\end{aligned}
$$

and $\int_{b}^{+\infty} \Delta_{\alpha}[f](t) \Delta_{\alpha} t=-f(b)$, it follows that

$$
\int_{a}^{b} \Delta_{\alpha}[f](t) \Delta_{\alpha} t=\int_{a}^{+\infty} f(t) \Delta_{\alpha} t-\int_{b}^{+\infty} f(t) \Delta_{\alpha} t=f(b)-f(a) .
$$

Corollary 2 ( $\alpha$-forward integration by parts). Let $f, g: I \rightarrow \mathbb{R}$. If $f g$ and $f \Delta_{\alpha}[g]$ are $\alpha$-forward integrable on $[a, b]$, then

$$
\int_{a}^{b} f(t) \Delta_{\alpha}[g](t) \Delta_{\alpha} t=\left.f(t) g(t)\right|_{a} ^{b}-\int_{a}^{b} \Delta_{\alpha}[f](t) g(t+\alpha) \Delta_{\alpha} t
$$

Proof. Since $\Delta_{\alpha}[f g](t)=\Delta_{\alpha}[f](t) g(t+\alpha)+f(t) \Delta_{\alpha}[g](t)$, then

$$
\begin{aligned}
\int_{a}^{b} f(t) \Delta_{\alpha}[g](t) \Delta_{\alpha} t & =\int_{a}^{b}\left(\Delta_{\alpha}[f g](t)-\Delta_{\alpha}[f](t) g(t+\alpha)\right) \Delta_{\alpha} t \\
& =\int_{a}^{b} \Delta_{\alpha}[f g](t) \Delta_{\alpha} t-\int_{a}^{b} \Delta_{\alpha}[f](t) g(t+\alpha) \Delta_{\alpha} t \\
& =\left.f(t) g(t)\right|_{a} ^{b}-\int_{a}^{b} \Delta_{\alpha}[f](t) g(t+\alpha) \Delta_{\alpha} t
\end{aligned}
$$

Remark 1. Our study of the Nörlund sum is in agreement with the Hahn quantum calculus [2, 3, 5]. In [4] $\int_{a}^{b} f(t) \Delta_{\alpha} t=\alpha[f(a)+f(a+\alpha)+\cdots+f(b-\alpha)]$ for $a<$ $b$ such that $b-a \in \alpha \mathbb{Z}, \alpha \in \mathbb{R}^{+}$. In contrast with [4], our definition is valid for any two real points $a, b$ and not only for those points belonging to the time scale $\alpha \mathbb{Z}$. The definitions (only) coincide if function $f$ is $\alpha$-forward integrable on $[a, b]$. 
Similarly, we introduce the $\beta$-backward integral.

Definition 2. Let $I$ be an interval of $\mathbb{R}$ such that $a, b \in I$ with $a<b$ and inf $I=-\infty$. For $f: I \rightarrow \mathbb{R}$ and $\beta>0$ we define the $\beta$-backward integral of $f$ from $a$ to $b$ by

$$
\int_{a}^{b} f(t) \nabla_{\beta} t=\int_{-\infty}^{b} f(t) \nabla_{\beta} t-\int_{-\infty}^{a} f(t) \nabla_{\beta} t
$$

where $\int_{-\infty}^{x} f(t) \nabla_{\beta} t=\beta \sum_{k=0}^{+\infty} f(x-k \beta)$, provided the series converges at $x=a$ and $x=b$. In that case, $f$ is called $\beta$-backward integrable on $[a, b]$. We say that $f$ is $\beta$-backward integrable over $I$ if it is $\beta$-backward integrable for all $a, b \in I$.

The $\beta$-backward Nörlund sum has similar results and properties as the $\alpha$-forward Nörlund sum. In particular, the $\beta$-backward integral is the inverse operator of $\nabla_{\beta}$.

\section{The $\alpha, \beta$-symmetric Nörlund sum}

We define the $\alpha, \beta$-symmetric integral as a linear combination of the $\alpha$-forward and the $\beta$-backward integrals.

Definition 3. Let $f: \mathbb{R} \rightarrow \mathbb{R}$ and $a, b \in \mathbb{R}, a<b$. If $f$ is $\alpha$-forward and $\beta$-backward integrable on $[a, b], \alpha, \beta \geq 0$ with $\alpha+\beta>0$, then we define the $\alpha, \beta$-symmetric integral of $f$ from $a$ to $b$ by

$$
\int_{a}^{b} f(t) d_{\alpha, \beta} t=\frac{\alpha}{\alpha+\beta} \int_{a}^{b} f(t) \Delta_{\alpha} t+\frac{\beta}{\alpha+\beta} \int_{a}^{b} f(t) \nabla_{\beta} t .
$$

Function $f$ is $\alpha, \beta$-symmetric integrable if it is $\alpha, \beta$-symmetric integrable for all $a, b \in \mathbb{R}$.

Remark 2. Note that if $\alpha \in \mathbb{R}^{+}$and $\beta=0$, then $\int_{a}^{b} f(t) d_{\alpha, \beta} t=\int_{a}^{b} f(t) \Delta_{\alpha} t$ and we do not need to assume in Definition 3 that $f$ is $\beta$-backward integrable; if $\alpha=0$ and $\beta \in \mathbb{R}^{+}$, then $\int_{a}^{b} f(t) d_{\alpha, \beta} t=\int_{a}^{b} f(t) \nabla_{\beta} t$ and we do not need to assume that $f$ is $\alpha$-forward integrable.

Example 1. Let $f(t)=1 / t^{2}$. Then $\int_{1}^{3} \frac{1}{t^{2}} d_{2,2} t=\frac{10}{9}$.

The $\alpha, \beta$-symmetric integral has the following properties:

Theorem 6. Let $f, g: \mathbb{R} \rightarrow \mathbb{R}$ be $\alpha, \beta$-symmetric integrable on $[a, b]$. Let $c \in[a, b]$ and $k \in \mathbb{R}$. Then,

1. $\int_{a}^{a} f(t) d_{\alpha, \beta} t=0$ 
2. $\int_{a}^{b} f(t) d_{\alpha, \beta} t=\int_{a}^{c} f(t) d_{\alpha, \beta} t+\int_{c}^{b} f(t) d_{\alpha, \beta}$, when the integrals exist;

3. $\int_{a}^{b} f(t) d_{\alpha, \beta} t=-\int_{b}^{a} f(t) d_{\alpha, \beta} t$;

4. $k f$ is $\alpha, \beta$-symmetric integrable on $[a, b]$ and $\int_{a}^{b} k f(t) d_{\alpha, \beta} t=k \int_{a}^{b} f(t) d_{\alpha, \beta} t$;

5. $f+g$ is $\alpha, \beta$-symmetric integrable on $[a, b]$ and

$$
\int_{a}^{b}(f+g)(t) d_{\alpha, \beta} t=\int_{a}^{b} f(t) d_{\alpha, \beta} t+\int_{a}^{b} g(t) d_{\alpha, \beta} t ;
$$

6. $f g$ is $\alpha, \beta$-symmetric integrable on $[a, b]$ provided $g$ is a nonnegative function.

Proof. These results are easy consequences of the $\alpha$-forward and $\beta$-backward integral properties.

The next result follows immediately from Theorem 3 and the corresponding $\beta$ backward version.

Theorem 7. Let $f: \mathbb{R} \rightarrow \mathbb{R}$ and $p>1$. If $|f|$ is symmetric $\alpha, \beta$-integrable on $[a, b]$, then $|f|^{p}$ is also $\alpha, \beta$-symmetric integrable on $[a, b]$.

Theorem 8. Let $f, g: \mathbb{R} \rightarrow \mathbb{R}$ be $\alpha, \beta$-symmetric integrable functions on $[a, b], \mathscr{A}:=$ $\left\{a+k \alpha: k \in \mathbb{N}_{0}\right\}$ and $\mathscr{B}:=\left\{b-k \beta: k \in \mathbb{N}_{0}\right\}$. For $b \in \mathscr{A}$ and $a \in \mathscr{B}$ one has:

1. if $|f(t)| \leqslant g(t)$ for all $t \in \mathscr{A} \cup \mathscr{B}$, then $\left|\int_{a}^{b} f(t) d_{\alpha, \beta} t\right| \leqslant \int_{a}^{b} g(t) d_{\alpha, \beta} t$;

2. if $f(t) \geqslant 0$ for all $t \in \mathscr{A} \cup \mathscr{B}$, then $\int_{a}^{b} f(t) d_{\alpha, \beta} t \geqslant 0$;

3. if $g(t) \geqslant f(t)$ for all $t \in \mathscr{A} \cup \mathscr{B}$, then $\int_{a}^{b} g(t) d_{\alpha, \beta} t \geqslant \int_{a}^{b} f(t) d_{\alpha, \beta} t$.

Proof. It follows from Theorem 4 and Corollary 1 and the corresponding $\beta$ backward versions.

In Theorem 9 we assume that $a, b \in \mathbb{R}$ with $b \in \mathscr{A}:=\left\{a+k \alpha: k \in \mathbb{N}_{0}\right\}$ and $a \in \mathscr{B}:=\left\{b-k \beta: k \in \mathbb{N}_{0}\right\}$, where $\alpha, \beta \in \mathbb{R}_{0}^{+}, \alpha+\beta \neq 0$.

Theorem 9 (Mean value theorem). Let $f, g: \mathbb{R} \rightarrow \mathbb{R}$ be bounded and $\alpha, \beta$-symmetric integrable on $[a, b]$ with $g$ nonnegative. Let $m$ and $M$ be the infimum and the supremum, respectively, of function $f$. Then, there exists a real number $K$ satisfying the inequalities $m \leqslant K \leqslant M$ such that $\int_{a}^{b} f(t) g(t) d_{\alpha, \beta} t=K \int_{a}^{b} g(t) d_{\alpha, \beta} t$.

Proof. Since $m \leqslant f(t) \leqslant M$ for all $t \in \mathbb{R}$ and $g(t) \geqslant 0$, then $m g(t) \leqslant f(t) g(t) \leqslant$ $M g(t)$ for all $t \in \mathscr{A} \cup \mathscr{B}$. All functions $m g, f g$ and $M g$ are $\alpha, \beta$-symmetric integrable on $[a, b]$. By Theorems 6 and $8, m \int_{a}^{b} g(t) d_{\alpha, \beta} t \leqslant \int_{a}^{b} f(t) g(t) d_{\alpha, \beta} t \leqslant$ $M \int_{a}^{b} g(t) d_{\alpha, \beta} t$. If $\int_{a}^{b} g(t) d_{\alpha, \beta} t=0$, then $\int_{a}^{b} f(t) g(t) d_{\alpha, \beta} t=0$; if $\int_{a}^{b} g(t) d_{\alpha, \beta} t>0$, then $m \leqslant \frac{\int_{a}^{b} f(t) g(t) d_{\alpha, \beta} t}{\int_{a}^{b} g(t) d_{\alpha, \beta} t} \leqslant M$. Therefore, the middle term of these inequalities is equal to a number $K$, which yields the intended result. 


\section{$4 \alpha, \beta$-Symmetric Integral Inequalities}

Inspired in the work by Agarwal et al. [1], we now present $\alpha, \beta$-symmetric versions of Hölder, Cauchy-Schwarz and Minkowski inequalities. As before, we assume that $a, b \in \mathbb{R}$ with $b \in \mathscr{A}:=\left\{a+k \alpha: k \in \mathbb{N}_{0}\right\}$ and $a \in \mathscr{B}:=\left\{b-k \beta: k \in \mathbb{N}_{0}\right\}$, where $\alpha, \beta \in \mathbb{R}_{0}^{+}, \alpha+\beta \neq 0$.

Theorem 10 (Hölder's inequality). Let $f, g: \mathbb{R} \rightarrow \mathbb{R}$ and $a, b \in \mathbb{R}$ with $a<b$. If $|f|$ and $|g|$ are $\alpha, \beta$-symmetric integrable on $[a, b]$, then

$$
\int_{a}^{b}|f(t) g(t)| d_{\alpha, \beta} t \leqslant\left(\int_{a}^{b}|f(t)|^{p} d_{\alpha, \beta} t\right)^{\frac{1}{p}}\left(\int_{a}^{b}|g(t)|^{q} d_{\alpha, \beta} t\right)^{\frac{1}{q}},
$$

where $p>1$ and $q=p /(p-1)$.

Proof. For $\alpha, \beta \in \mathbb{R}_{0}^{+}, \alpha+\beta \neq 0$, the following inequality holds: $\alpha^{\frac{1}{p}} \beta^{\frac{1}{q}} \leqslant \frac{\alpha}{p}+\frac{\beta}{q}$. Without loss of generality, suppose that $\left(\int_{a}^{b}|f(t)|^{p} d_{\alpha, \beta} t\right)\left(\int_{a}^{b}|g(t)|^{q} d_{\alpha, \beta} t\right) \neq 0$ (note that both integrals exist by Theorem 7). Set $\xi(t)=|f(t)|^{p} / \int_{a}^{b}|f(\tau)|^{p} d_{\alpha, \beta} \tau$ and $\gamma(t)=|g(t)|^{q} / \int_{a}^{b}|g(\tau)|^{q} d_{\alpha, \beta} \tau$. Since both functions $\alpha$ and $\beta$ are symmetric $\alpha, \beta$-integrable on $[a, b]$, then (1) holds:

$$
\begin{aligned}
& \int_{a}^{b} \frac{|f(t)|}{\left(\int_{a}^{b}|f(\tau)|^{p} d_{\alpha, \beta} \tau\right)^{\frac{1}{p}}} \frac{|g(t)|}{\left(\int_{a}^{b}|g(\tau)|^{q} d_{\alpha, \beta} \tau\right)^{\frac{1}{q}}} d_{\alpha, \beta} t=\int_{a}^{b} \xi(t)^{\frac{1}{p}} \gamma(t)^{\frac{1}{q}} d_{\alpha, \beta} t \\
& \quad \leqslant \int_{a}^{b}\left(\frac{\xi(t)}{p}+\frac{\gamma(t)}{q}\right) d_{\alpha, \beta} t \\
& \quad=\frac{1}{p} \int_{a}^{b}\left(\frac{|f(t)|^{p}}{\int_{a}^{b}|f(\tau)|^{p} d_{\alpha, \beta} \tau}\right) d_{\alpha, \beta} t+\frac{1}{q} \int_{a}^{b}\left(\frac{|g(t)|^{q}}{\int_{a}^{b}|g(\tau)|^{q} d_{\alpha, \beta} \tau}\right) d_{\alpha, \beta} t=1 .
\end{aligned}
$$

The particular case $p=q=2$ of (1) gives the Cauchy-Schwarz inequality.

Corollary 3 (Cauchy-Schwarz's inequality). Let $f, g: \mathbb{R} \rightarrow \mathbb{R}$ and $a, b \in \mathbb{R}$ with $a<b$. If $f$ and $g$ are $\alpha, \beta$-symmetric integrable on $[a, b]$, then

$$
\int_{a}^{b}|f(t) g(t)| d_{\alpha, \beta} t \leqslant \sqrt{\left(\int_{a}^{b}|f(t)|^{2} d_{\alpha, \beta} t\right)\left(\int_{a}^{b}|g(t)|^{2} d_{\alpha, \beta} t\right)} .
$$

We prove the Minkowski inequality using Hölder's inequality.

Theorem 11 (Minkowski's inequality). Let $f, g: \mathbb{R} \rightarrow \mathbb{R}$ and $a, b, p \in \mathbb{R}$ with $a<b$ and $p>1$. If $f$ and $g$ are $\alpha, \beta$-symmetric integrable on $[a, b]$, then

$$
\left(\int_{a}^{b}|f(t)+g(t)|^{p} d_{\alpha, \beta} t\right)^{\frac{1}{p}} \leqslant\left(\int_{a}^{b}|f(t)|^{p} d_{\alpha, \beta} t\right)^{\frac{1}{p}}+\left(\int_{a}^{b}|g(t)|^{p} d_{\alpha, \beta} t\right)^{\frac{1}{p}} .
$$


Proof. One has

$$
\begin{aligned}
\int_{a}^{b} \mid f(t) & +\left.g(t)\right|^{p} d_{\alpha, \beta} t=\int_{a}^{b}|f(t)+g(t)|^{p-1}|f(t)+g(t)| d_{\alpha, \beta} t \\
& \leqslant \int_{a}^{b}|f(t)||f(t)+g(t)|^{p-1} d_{\alpha, \beta} t+\int_{a}^{b}|g(t)||f(t)+g(t)|^{p-1} d_{\alpha, \beta} t .
\end{aligned}
$$

Applying Hölder's inequality (Theorem 10 with $q=p /(p-1)$, we obtain

$$
\begin{aligned}
& \int_{a}^{b}|f(t)+g(t)|^{p} d_{\alpha, \beta} t \leqslant\left(\int_{a}^{b}|f(t)|^{p} d_{\alpha, \beta} t\right)^{\frac{1}{p}}\left(\int_{a}^{b}|f(t)+g(t)|^{(p-1) q} d_{\alpha, \beta} t\right)^{\frac{1}{q}} \\
+ & \left(\int_{a}^{b}|g(t)|^{p} d_{\alpha, \beta} t\right)^{\frac{1}{p}}\left(\int_{a}^{b}|f(t)+g(t)|^{(p-1) q} d_{\alpha, \beta} t\right)^{\frac{1}{q}} \\
= & {\left[\left(\int_{a}^{b}|f(t)|^{p} d_{\alpha, \beta} t\right)^{\frac{1}{p}}+\left(\int_{a}^{b}|g(t)|^{p} d_{\alpha, \beta} t\right)^{\frac{1}{p}}\right]\left(\int_{a}^{b}|f(t)+g(t)|^{(p-1) q} d_{\alpha, \beta} t\right)^{\frac{1}{q}} . }
\end{aligned}
$$

Therefore,

$$
\frac{\int_{a}^{b}|f(t)+g(t)|^{p} d_{\alpha, \beta} t}{\left(\int_{a}^{b}|f(t)+g(t)|^{(p-1) q} d_{\alpha, \beta} t\right)^{\frac{1}{q}}} \leqslant\left(\int_{a}^{b}|f(t)|^{p} d_{\alpha, \beta} t\right)^{\frac{1}{p}}+\left(\int_{a}^{b}|g(t)|^{p} d_{\alpha, \beta} t\right)^{\frac{1}{p}} .
$$

Our $\alpha, \beta$-symmetric calculus is more general than the standard $h$-calculus. In particular, all our results give, as corollaries, results in the classical quantum $h$ calculus by choosing $\alpha=h>0$ and $\beta=0$.

Acknowledgements Work supported by FEDER and Portuguese funds, COMPETE reference FCOMP-01-0124-FEDER-022690, and CIDMA and FCT, project PEst-C/MAT/UI4106/2011. Brito da Cruz is also supported by FCT through the Ph.D. fellowship SFRH/BD/33634/2009.

\section{References}

1. R. Agarwal, M. Bohner and A. Peterson, Inequalities on time scales: a survey, Math. Inequal. Appl. 4 (2001), no. 4, 535-557.

2. K. A. Aldwoah, Generalized time scales and associated difference equations, Ph.D. thesis, Cairo University, 2009.

3. A. M. C. Brito da Cruz, N. Martins and D. F. M. Torres, Higher-order Hahn's quantum variational calculus, Nonlinear Anal. 75 (2012), no. 3, 1147-1157. arXiv:1101.3653

4. V. Kac and P. Cheung, Quantum calculus, Springer, New York, 2002.

5. A. B. Malinowska and D. F. M. Torres, The Hahn quantum variational calculus, J. Optim. Theory Appl. 147 (2010), no. 3, 419-442. arXiv:1006.3765

6. B. S. Thomson, Symmetric properties of real functions, Dekker, New York, 1994. 